\title{
ON PERTURBATIONS OF M-ACCRETIVE OPERATORS IN BANACH SPACES
}

\author{
NORIMICHI HIRANO AND A. K. KALINDE
}

(Communicated by Palle E. T. Jorgensen)

\begin{abstract}
In this paper, we consider the solvability of nonlinear equations of the form$$
A u+C u \ni p
$$

where $A$ is an m-accretive operator on a Banach space $X, C$ is a mapping on $X$ and $p \in X$
\end{abstract}

\section{INTRODUCTION}

Let $X$ be a real Banach space, $A: X \supset D(A) \rightarrow X$ be an m-accretive operator, $C: X \supset \overline{D(A)} \rightarrow X$ is a continuous mapping and $p \in X$. In the last decade, the solvability of the nonlinear equation

$$
A u+C u \ni p
$$

has been studied by Hirano [3], Kartsatos [4, 6], Morales [8], and other authors (cf. Kartsatos [5] for further references). The abstract results for $(\mathrm{P})$ are applied to partial differential equations (cf. [5]).

Our purpose in this paper is to consider the solvability of problem $(\mathrm{P})$ in the case that $C: X \supset D(A) \rightarrow X$ is not necessarily continuous. The results for noncontinuous cases can be applied to nonlinear boundary value problems (cf. the Example below).

In what follows, $X$ stands for a real Banach space with norm $\|\cdot\|$, and $J$ stands for the normalized duality mapping. For each $x \in X^{*}$ and $x \in X$, we denote by $\left\langle x, x^{*}\right\rangle$ the value of $x^{*}$ at $x$. An operator $A: X \supset D(A) \rightarrow 2^{X}$ is said to be accretive if for every $x, y \in D(A)$, there exists $j \in J(x-y)$ such that

$$
\langle u-v, j\rangle \geq 0, \quad \text { for all } u \in A x, v \in A y .
$$

An accretive operator is said to be m-accretive if $R(I+\lambda A)=X$ for all $\lambda \in$ $(0, \infty)$. For an m-accretive operator $A$, the resolvent $J_{\lambda}: X \rightarrow D(A)$ is defined by $J_{\lambda}=(I+\lambda A)^{-1}$ for all $\lambda \in(0, \infty)$. We denote by $A_{\lambda}$ the operator defined as $A_{\lambda}=(1 / \lambda)\left(I-J_{\lambda}\right)$ for $\lambda \in(0, \infty)$. It is easy to see that $A_{\lambda} x \in A J_{\lambda} x$ for $x \in X$.

Received by the editors May 17, 1994 and, in revised form, October 10, 1994.

1991 Mathematics Subject Classification. Primary 47H17; Secondary 47H05, 47B07, 47H10.

Key words and phrases. Perturbations of $\mathrm{m}$-accretive operators, compact resolvent, condensing mapping. 
For each $x \in D(A)$, we set $|A x|=\inf \{\|v\|: v \in A x\}$. For each bounded set $S \subset X$, the measure of noncompactness $\alpha(S)$ of $S$ is defined by

$$
\begin{aligned}
\alpha(S)=\inf \{\delta>0: & S \text { can be covered with a finite } \\
& \text { number of sets of diameter less than } \delta\} .
\end{aligned}
$$

A continuous mapping $F: X \supset D(F) \rightarrow X$ is said to be condensing if, for every bounded noncompact subset $S$ of $D(F), F(S)$ is bounded and $\alpha(F(S))<\alpha(S)$ (cf. [7]). It is obvious from the definition that $F$ is condensing if $F$ is compact or Lipschitz continuous with Lipschitz constant $k<1$. It is also easy to see that the sum of a condensing mapping and a compact mapping is a condensing mapping. A mapping $F: X \supset D(F) \rightarrow X$ is said to be bounded if $F$ maps each bounded set of $D(F)$ to bounded sets of $X$. We denote by $B_{r}(x)$ the open ball with center at $x$ and radius $r>0$. For each subset $D$ of $X$, we denote by $\partial D$ the boundary of $D$. We denote by $\Gamma$ the set of all functions $\beta: R^{+} \rightarrow R^{+}$such that $\beta(r) \rightarrow 0$ as $r \rightarrow \infty$. We first consider the case that the m-accretive operator $A$ has a compact resolvent.

Theorem 1. Let $A: X \supset D(A) \rightarrow 2^{X}$ be m-accretive with $(A+I)^{-1}$ compact. Let $C: X \supset D(A) \rightarrow X$ be bounded and satisfy that $C(I+\lambda A)^{-1}: X \rightarrow X$ is condensing for some $\lambda \in(0,1]$. Let $p \in X$ and assume that there exist positive constants $b, r$ and $z \in D(A)$ satisfying that $\|z\|<b$ and for every $x \in D(A)$ with $\|x\| \geq b$,

$$
\langle u+C x-p, j\rangle \geq 0
$$

for all $u \in A x$ and all $j \in J(x-z)$. Then $p \in R(A+C)$.

Proof. Let $\lambda \in(0,1]$ be such that $C(I+\lambda A)^{-1}$ is condensing. Let $y \in X$ satisfy the equality

$$
A_{\lambda} y+C J_{\lambda} y=p .
$$

Then recalling that $A_{\lambda} y \in A J_{\lambda} y$, we have that $x=J_{\lambda} y$ is a solution of the problem (P). Then we seek a solution of (1.1). Since $A_{\lambda} y=(1 / \lambda)\left(I-J_{\lambda}\right) y$, equation (1.1) can be rewritten as

$$
y=\lambda p+J_{\lambda} y-\lambda C J_{\lambda} y .
$$

We now define a mapping $T: X \rightarrow X$ by

$$
T y=\lambda p+J_{\lambda} y-\lambda C J_{\lambda} y .
$$

Then by (1.2), the solution of (1.1) is a fixed point of the mapping $T$. Since $J_{\lambda}$ is compact and $C J_{\lambda}$ is condensing, we have that $T$ is condensing on $X$. Here we fix $w \in X$ such that $w \in z+\lambda A z$. Since $C$ is bounded, we can choose $M>0$ such that

$$
\sup _{x \in D(A),\|x\| \leq b}\|\lambda p+x-\lambda C x-w\|<M .
$$

We choose $r>0$ so large that $r>\max \{(1+\lambda) b,\|w\|\}$ and

$$
r-2 b-\|w\|>M \text {. }
$$

By Theorem 6.3.2 of [7], $T$ possesses a fixed point in $\overline{B_{r}(0)}$ if

$$
T(y)-w \neq \alpha(y-w), \quad \text { for all } y \in \partial B_{r}(0) \quad \text { and } \alpha>1 .
$$


Suppose that $y \in \partial B_{r}(0)$ and $T(y)-w=\alpha(y-w)$ for some $\alpha>1$. Then we have by (1.3) that

$$
\alpha(y-w)+w=\lambda p+J_{\lambda} y-\lambda C J_{\lambda} y .
$$

We put $x=J_{\lambda} y$. Then $x+\lambda A x \ni y$. Let $v \in A x$ such that $x+\lambda v=y$. Then

$$
\alpha x+\alpha(\lambda v-w)=\lambda p+x-\lambda C x-w .
$$

Now suppose that $\|x\|<b$; then by (1.4),

$$
\|\lambda p+x-\lambda C x-w\|<M .
$$

On the other hand, noting that $\lambda\|v\|=\|y-x\| \geq r-b$,

$$
\begin{aligned}
\|\alpha x+\alpha(\lambda v-w)\| & \geq \alpha \lambda\|v\|-\alpha(\|x\|+\|w\|) \\
& \geq \alpha((r-b)-b-\|w\|) \\
& \geq r-2 b-\|w\|>M .
\end{aligned}
$$

This contradicts (1.8). Therefore we have that $\|x\| \geq b$

On the other hand, recalling that $v \in A x$, we have by the accretivity of $A$ that there exists $j \in J(x-z)$ and

$$
\alpha\langle x+\lambda v-w, j\rangle>\langle x+\lambda v-w, j\rangle \geq\|x-z\|^{2}>0 .
$$

Then by (1.8), we have

$$
\langle\lambda p+x-\lambda C x-w, j\rangle>\langle x+\lambda v-w, j\rangle .
$$

Thus we find

$$
\langle v+C x-p, j\rangle<0 .
$$

Since $\|x\| \geq b,(1.10)$ contradicts the condition $(*)$. Therefore (1.6) holds and then (1.1) has a solution. This completes the proof.

Theorem 2. Let $A: X \supset D(A) \rightarrow 2^{X}$ be m-accretive with $(A+I)^{-1}$ compact. Let $C: X \supset \overline{D(A)} \rightarrow X$ be continuous and bounded, and let $p \in X$. Assume that there exist positive constants $b, r$ and $z \in D(A)$ satisfying that $\|z\|<b$ and, for every $x \in D(A)$ with $\|x\| \geq b,(*)$ holds for all $u \in A x$ and all $j \in J(x-z)$. Then $p \in R(A+C)$.

Proof. Since $C: \overline{D(A)} \rightarrow X$ is bounded and continuous, we have that $C(I+A)^{-1}$ is compact. Then the assertion follows from Theorem 1.

We can treat the case that $C$ is not defined on $\overline{D(A)}$ if the dual space $X^{*}$ of $X$ is uniformly convex:

Theorem $2^{\prime}$. Let $X^{*}$ be uniformly convex. Let $A: X \supset D(A) \rightarrow 2^{X}$ be $m$-accretive with $(A+I)^{-1}$ compact. Let $C: X \supset D(A) \rightarrow X$ be continuous and bounded, and let $p \in X$. Assume that there exist positive constants $b, r$ and $z \in D(A)$ satisfying that $\|z\|<b$ and, for every $x \in D(A)$ with $\|x\| \geq b,(*)$ holds for all $u \in A x$ and, for $j=J(x-z)$. Then $p \in R(A+C)$.

Proof. It is sufficient to show that $C(I+A)^{-1}$ is compact. Let $\left\{x_{n}\right\} \subset X$ be a bounded sequence and put $y_{n}=(I+A)^{-1} x_{n}$ for $n \geq 1$. We may assume that $x_{n}$ converges weakly to $x \in X$ and $y_{n}$ converges strongly to $y \in X$. Then since the m-accretive operator $A$ is demiclosed (cf. Proposition 3.5 of Barbu [1]), we find that $y \in D(A)$ and $x \in A y$. Then by the continuity of $C$ on $D(A)$, we find that $C y_{n} \rightarrow C y$. That is, $C(I+A)^{-1}$ is compact. 
Remark. Theorem 2 (Theorem $2^{\prime}$ ) should be compared with Theorem 5 of [6]. It is known that the duality mapping $J$ is single valued if $X^{*}$ is strictly convex. If $J$ is single valued, then condition $(*)$ of Kartsatos [6] is the condition $(*)$ with $z=0$. We also note that as we have seen in the proof of Theorem $2^{\prime}$, our argument does not require the mapping $C$ to be defined on $\overline{D(A)}$ if the m-accretive operator $A$ is demiclosed. The known results so far demand $C$ to be defined and continuous on $\overline{D(A)}$.

We next treat the case that $C$ is not bounded.

Theorem 3. Let $A: X \supset D(A) \rightarrow 2^{X}$ be m-accretive with $(A+I)^{-1}$ compact. Let $C: X \supset D(A) \rightarrow X$ satisfy that $C(I+\lambda A)^{-1}: X \rightarrow X$ is condensing for some $\lambda \in(0,1]$, and let $p \in X$. Assume that there exist positive constants $b, r$ and $z \in D(A)$ satisfying that $\|z\|<b, A z$ is bounded and, for every $x \in D(A)$ with $\max \{\|x\|, \lambda|A x|\} \geq b,(*)$ holds for all $u \in A x$ and all $j \in J(x-z)$. Then $p \in R(A+C)$.

Proof. We put $M=\sup \{\|v\|: v \in A z\}$. Let $r>0$ be so large that

$$
r>b+\lambda \max \{b, M\} \text {. }
$$

Let $x, y, v$ and $z$ be as in the proof of Theorem 1. Then since $\|y\|=r$ and $y=x+\lambda v$, we find from (1.11) that

$$
\|x\|>b \quad \text { or } \quad\|v\|>\max \{b, M\} .
$$

This implies that $x \neq z$. Then by (1.9), we find that (1.10) holds. On the other hand, it follows from $(1.12)$ that $(*)$ holds. This is a contradiction and the proof is complete.

We next consider the solvability of $(\mathrm{P})$ under a condition introduced in Guan and Karsatos [2]. In the next result, we impose the following condition on $C$ :

(c) If $\left\{x_{n}\right\} \subset X$ is a convergent sequence such that $x=\lim _{n \rightarrow \infty} x_{n}$ and there exists a bounded sequence $\left\{v_{n}\right\} \subset X$ with $v_{n} \in A x_{n}$ for $n \geq 1$, then $C x=$ $\lim _{n \rightarrow \infty} C x_{n}$.

Theorem 4. Let $A: X \supset D(A) \rightarrow 2^{X}$ be m-accretive with $(A+I)^{-1}$ compact. Let $C: X \supset D(A) \rightarrow X$ be bounded, satisfy (c) and satisfy that $C(I+\lambda A)^{-1}: X \rightarrow X$ is condensing for some $\lambda \in(0,1]$. Let $S \subset X$ be such that for every $p \in S$ there exists $K(p)>0, \beta=\beta_{s} \in \Gamma$ and $z \in D(A)$ such that

$$
\langle v+C x-p, j\rangle \geq-K(p)-\beta(\|x\|)\|x\|,
$$

for all $x \in D(A)$ with $\|x\|$ sufficiently large, all $v \in A x$ and for all $j \in J(x-z)$. Then $S \subset \overline{R(A+B)}$ and int $S \subset R(A+B)$.

Proof. Let $p \in S$. We set

$$
C_{n} x=C x+(1 / n)(x-z) \text { for } x \in D(A) \text { and } n \geq 1 .
$$

Then $C_{n}$ is bounded. Since $(I+A)^{-1}$ is compact, we can see that $C_{n}(I+\lambda A)^{-1}=$ $C(I+\lambda A)^{-1}+(1 / n)(I+\lambda A)^{-1}-(1 / n) z$ is condensing. From $(* *)$, we have that for each $n>1$,

$$
\left\langle v+C_{n} x-p, j\right\rangle \geq(1 / n)\|x-z\|^{2}-K(p)-\beta(\|x\|)\|x\|
$$


for all $x \in D(A)$ with $\|x\|$ sufficiently large, all $v \in A x$ and for all $j=j_{x, s} \in J(x-z)$. It then follows that for each $n \geq 1$, there exists $b_{n}>0$ such that

$$
\left\langle v+C_{n} x-p, j\right\rangle \geq 0
$$

for all $x \in D(A)$ with $\|x\| \geq b_{n}$, all $v \in A x$ and for all $j=j_{x, s} \in J(x-z)$. Then by Theorem 1 , we find that $p \in R\left(A+C_{n}\right)$ for all $n \geq 1$. That is, there exist sequences $\left\{x_{n}\right\} \subset X$ and $\left\{v_{n}\right\} \subset X$ such that $v_{n} \in A x_{n}$ and

$$
v_{n}+C x_{n}+\frac{1}{n}\left(x_{n}-z\right)=p \text { for all } n \geq 1 .
$$

If $\left\{x_{n}\right\}$ contains a bounded subsequence, then from (1.13), we have that $p \in$ $\overline{R(A+C)}$. We now assume that $\lim _{n \rightarrow \infty}\left\|x_{n}\right\|=\infty$. Then we have by (**) and (1.13) that

$$
(1 / n)\left\|x_{n}-z\right\|^{2} \leq K(p)+\beta\left(\left\|x_{n}\right\|\right)\left\|x_{n}\right\|
$$

for $n$ sufficiently large. Then since

$$
\lim _{n \rightarrow \infty}\left\|x_{n}\right\| /\left\|x_{n}-z\right\|=1
$$

and

$$
\lim _{n \rightarrow \infty} \beta\left(\left\|x_{n}\right\|\right)=0,
$$

we find that $\lim _{n \rightarrow \infty}(1 / n)\left\|x_{n}-z\right\|=0$. It then follows from (1.13) that $p \in$ $\overline{R(A+C)}$. Thus we have shown that $S \subset \overline{R(A+C)}$.

We next show int $S \subset R(A+C)$. Suppose that $p \in \operatorname{int} S$ and let $\left\{x_{n}\right\}$ be the sequence of solutions of (1.13). Suppose that there exists a bounded subsequence $\left\{x_{m}\right\}$ of $\left\{x_{n}\right\}$. By (1.13), we find that

$$
x_{m}=(I+A)^{-1}\left(x_{m}+p-\frac{1}{n}\left(x_{m}-z\right)-C x_{m}\right) .
$$

Since $\left\{x_{m}\right\}$ is bounded, we have by the hypothesis that $\left\{C x_{m}\right\}$ is bounded. Then by (1.14), we have that $\left\{x_{m}\right\}$ is precompact. Then we may assume that $x_{n}$ converges to $x \in X$. It also follows from (1.13) that $\left\{v_{n}\right\}$ is bounded. Then by (c), $\lim _{n \rightarrow \infty} C x_{n}=C x$. This implies that $v_{n} \rightarrow v \in X$ and then $v \in A x$ (cf. Proposition 3.4 of [1]). Thus we find that $A x+C x \ni p$. We next suppose that $\lim _{n \rightarrow \infty}\left\|x_{n}\right\|=\infty$. Let $j_{n} \in J\left(x_{n}-z\right)$. We put

$$
\tilde{j}_{n}=j_{n} / \max \left\{\beta\left(\left\|x_{n}\right\|\right)\left\|x_{n}\right\|, 1\right\} \quad \text { for each } n \geq 1 \text {. }
$$

Since $\beta \in \Gamma$ we have that $\lim _{n \rightarrow \infty}\left\|\tilde{j}_{n}\right\|=\infty$. Since $p \in \operatorname{int} S$, there exists $r>0$ such that $B_{r}(p) \subset S$. Let $h \in B_{r}(0)$. Then since

$$
v_{n}+C x_{n}+\frac{1}{n}\left(x_{n}-z\right)-(p+h)=-h,
$$

we have by $(* *)$,

$$
\left\langle h, j_{n}\right\rangle \leq \beta\left(\left\|x_{n}\right\|\right)\left\|x_{n}\right\|+K(h+p)
$$

for each $n \geq 1$. Then

$$
\left\langle h, \tilde{j}_{n}\right\rangle \leq 1+K(h+p) \text { for all } n \geq 1 .
$$

Since $h \in B_{r}(0)$ is arbitrary, we have by the Banach-Steinhauss theorem that $\left\{\tilde{j}_{n}\right\}$ is bounded in $X^{*}$. This is a contradiction. Thus we have that $\left\{x_{n}\right\}$ is bounded in $X$ and then $A x+C x \ni p$. This completes the proof. 
Corollary 5. Let $A: X \supset D(A) \rightarrow 2^{X}$ be m-accretive with $(A+I)^{-1}$ compact. Let $C: X \supset \overline{D(A)} \rightarrow X$ be bounded and continuous. Let $S \subset X$ be such that for every $p \in S$ there exists $K(p)>0, \beta=\beta_{s} \in \Gamma$ and $z \in D(A)$ such that (**) holds for all $x \in D(A)$ with $\|x\|$ sufficiently large, all $v \in A x$ and for all $j \in J(x-z)$. Then $S \subset \overline{R(A+B)}$ and int $S \subset R(A+B)$.

Proof. Since $C$ is bounded and continuous, $C$ satisfies (c) and that $C(I+A)^{-1}$ is compact. Then the assertions follow from Theorem 4.

We next consider the case that the mapping $C(I+\lambda A)^{-1}$ is compact for some $\lambda>0$.

Theorem 6. Let $A: X \supset D(A) \rightarrow 2^{X}$ be m-accretive with $J_{1}=(A+I)^{-1}$ condensing. Let $C: X \supset D(A) \rightarrow X$ be bounded and satisfy that $C(I+\lambda A)^{-1}$ is compact for some $\lambda \in(0,1]$. Let $p \in X$ and assume that there exist positive constants $b, r$ and $z \in D(A)$ such that $\|z\|<b$ and, for every $x \in D(A)$ with $\|x\| \geq b,(*)$ holds for all $u \in A x$ and all $j \in J(x-z)$. Then $p \in R(A+C)$.

Proof. The proof of Theorem 6 is the same as that of Theorem 1. In fact, by the hypothesis, the mapping $T$ defined in the proof of Theorem 1 is condensing.

Theorem 7. Let $A: X \supset D(A) \rightarrow 2^{X}$ be m-accretive. Let $C: X \supset D(A) \rightarrow X$ be bounded and satisfy that $C(I+\lambda A)^{-1}$ is compact for some $\lambda \in(0,1]$. Let $p \in X$ and assume that there exist positive constants $b, r$ and $z \in D(A)$ such that $\|z\|<b$ and, for every $x \in D(A)$ with $\|x\| \geq b,(*)$ holds for all $u \in A x$ and all $j \in J(x-z)$. Then $p \in \overline{R(A+C)}$.

Proof. For each $n \geq 1$, we put $A(n)=A+(1 / n) I$. Then the resolvent $J_{1}(n)=$ $(I+A(n))^{-1}$ is Lipschitz mapping with Lipschitz constant $n /(1+n)$. That is, $J_{1}(n)$ is condensing. Then by applying Theorem 6 with $A$ replaced by $A(n)$, we find that there exist sequences $\left\{x_{n}\right\} \subset X$ and $\left\{v_{n}\right\} \subset X$ such that $v_{n} \in A x_{n}$ and

$$
v_{n}+\frac{1}{n} x_{n}+C x_{n}=p \text { for all } n \geq 1 \text {. }
$$

To show the assertion, it is sufficient to show that $\left\{x_{n}\right\}$ contains a subsequence such that $\lim _{n \rightarrow \infty} x_{n} / n=0$. If $\left\{x_{n}\right\}$ contains a bounded subsequence $\left\{x_{n}\right\}$, then $\lim _{m \rightarrow \infty} x_{m} / m=0$ and the assertion follows. Suppose that $\lim _{n \rightarrow \infty}\left\|x_{n}\right\|=\infty$. Then from (1.15) and $(*)$, we find that

$$
\left\langle-\frac{1}{n} x_{n}, j_{n}\right\rangle \geq 0
$$

for sufficiently large $n$, where $j_{n} \in J\left(x_{n}-z\right)$. Then it follows from the definition of $j_{n}$ that

$$
\frac{1}{n}\left\|x_{n}-z\right\| \leq \frac{1}{n}\|z\|
$$

for $n$ sufficiently large. Then we obtain that $\lim _{n \rightarrow \infty} x_{n} / n=0$ and the proof is complete.

Corollary 8. Let $A: X \supset D(A) \rightarrow 2^{X}$ be m-accretive and $C: X \supset D(A) \rightarrow X$ be compact. Let $p \in X$ and assume that there exist positive constants $b, r$ and $z \in D(A)$ such that $\|z\|<b$ and, for every $x \in D(A)$ with $\|x\| \geq b$, (*) holds for all $u \in T x$ and all $j \in J(x-z)$. Then $p \in \overline{R(A+C)}$. 
Proof. It is obvious that if $C$ is compact, then $C(I+A)^{-1}$ is compact. Then the assertion follows from Theorem 7 .

Remark. Corollary 5 and Corollary 8 should be compared with Theorem 4.1 of [2] and Theorem 3 of [6], respectively. We note that we do not know if the assertion of Theorem 4 holds in the case that $A$ is m-accretive, $C(I+\lambda A)^{-1}$ is compact for some $\lambda>0$ and $p \in X$ satisfies the condition $(* *)$.

Example. Let $\Omega \subset R^{N}$ be a bounded domain with a smooth boundary $\partial \Omega$. We consider the solvability of a nonlinear elliptic boundary value problem of the form

$$
\left\{\begin{array}{l}
-\sum_{i=1}^{N} \frac{\partial}{\partial x_{i}} a_{i}\left(\frac{\partial u}{\partial x_{i}}\right)+g\left(x, \frac{\partial u}{\partial x_{1}}, \ldots, \frac{\partial u}{\partial x_{N}}, u\right)=p(x) \text { on } \Omega \\
u(x)=0 \quad \text { on } \partial \Omega
\end{array}\right.
$$

Here we assume that $a_{i} \in C^{1}(R)$ is a function such that $0<\inf _{t \in R} a_{i}^{\prime}(t) \leq$ $\sup _{t \in R} a^{\prime}(t)<\infty$ for each $i=1, \ldots, N$. We define a nonlinear operator $A$ by

$$
A u=-\sum_{i=1}^{N} \frac{\partial}{\partial x_{i}} a_{i}\left(\frac{\partial u}{\partial x_{i}}\right) \quad \text { for } u \in D(A)=H^{2}(\Omega) \cap H_{0}^{1}(\Omega) .
$$

Then $A$ is an m-accretive operator on $X=L^{2}(\Omega)$ (cf. Barbu [1]). Let $p \in L^{2}(\Omega)$ and $g \in C\left(\Omega \times R^{N} \times R, R\right)$ satisfy that there exist positive constants $c_{1}, c_{2}$ and

$$
|g(x, s, t)| \leq c_{1}|t|+c_{2} \quad \text { for all } x \in \Omega, s \in R^{N} \text { and } t \in R,
$$

$$
\int_{\Omega}(g(x, D u(x), u(x))-p(x)) u(x) d x \geq 0
$$

for all $u \in D(A)$ with $\|u\|_{L^{2}} \geq b$, where $(D u)(x)=\left(\partial u / \partial x_{1}, \ldots, \partial u / \partial x_{N}\right)$ for $x \in \Omega$.

Here we put $(C u)(x)=g(x, D u(x), u(x))$ for $u \in D(A)$ and $x \in \Omega$. Then from $(g 1)$, we have that $C: X \supset D(A) \rightarrow X$ is bounded. From the assumption, the mapping $(I+A)^{-1}$ maps bounded sets of $L^{2}(\Omega)$ to bounded sets of $H^{2}(\Omega)$. Then recalling that $H^{2}(\Omega)$ is compactly embedded in $H^{1}(\Omega)$, we find that $C(I+A)^{-1}$ is compact in $L^{2}(\Omega)$. It is easy to see from the definition of $A$ and $(g 2)$ that $(*)$ holds. Then by Theorem 1, problem (BV) has a solution.

\section{REFERENCES}

1. V. Barbu Nonlinear semigroups and differential equations in Banach spaces, Noordhoff, Leyden, The Netherlands, 1976. MR 52:11666

2. Z. Guan and A. G. Kartsatos, Ranges of perturbed maximal monotone and m-accretive operators in Banach spaces, Trans. Amer. Math. Soc. 347 (1995), 2403-2435. MR 95i:47096

3. N. Hirano, Some surjectivity theorem for compact perturbations of accretive operators, Nonlinear Anal. TMA 8 (1984), 765-774. MR 86a:47054

4. A. G. Kartsatos, Mapping theorems involving ranges of sums of nonlinear operator, Nonlinear Anal. TMA 6 (1982), 271-278 MR 83k:47036

5. _ Recent results involving compact perturbations and compact resolvents of accretive operators in Banach spaces, Proceedings of the First World Congress of Nonlinear Analysts (Tampa, Florida, 1992), Walter De Gruyter (to appear). 
6. On compact perturbations and compact resolvents of nonlinear $m$-accretive operators in Banach spaces, Proc. Amer. Math. Soc. 119 (1993), 1189-1199. MR 94c:47076

7. E. Lloyd, Degree theory, Cambridge Univ. Press, New York, 1978. MR 58:12558

8. C. Morales, Remarks on compact perturbations of m-accretive operators, Nonlinear Anal. 16 (1991), 771-780. MR 92e:47118

Department of Mathematics, Faculty of Engineering, Yokohama National Univesity, Tokiwadai, Hodogayaku, Yokohama, Japan

E-mail address: hirano@math.sci.ynu.ac.jp

Faculty of Sciences, University of Fort Hare, P/Bag X1314 Alice 5700, Republic of Ciskei, South Africa 